\title{
Characterization and Control of Quantum Spin Chains and Rings
}

\author{
(Invited Paper) \\ Sophie G Schirmer*, Frank C Langbein ${ }^{\dagger}$ \\ * College of Science (Physics), Swansea University, \\ Singleton Park, Swansea, SA2 8PP, UK; Email: sgs29@swan.ac.uk \\ ${ }^{\dagger}$ School of Computer Science and Informatics, Cardiff University, \\ 5 The Parade, Cardiff, CF24 3AA, UK; Email: F.C.Langbein@cs.cardiff.ac.uk
}

\begin{abstract}
Information flow in quantum spin networks is considered. Two types of control - temporal bang-bang switching control and control by varying spatial degrees of freedom - are explored and shown to be effective in speeding up information transfer and increasing transfer fidelities. The control is modelbased and therefore relies on accurate knowledge of the system parameters. An efficient protocol for simultaneous identification of the coupling strength and the exact number of spins in a chain is presented.
\end{abstract}

\section{INTRODUCTION}

Nature, at a fundamental level, is governed by the laws of quantum mechanics. Until recently quantum phenomena were mostly studied by physicists but significant advances in theory and technology are increasingly pushing quantum phenomena into the realm of engineering, as building blocks for novel technologies and applications from chemistry to computing. Among the interesting applications are spin networks. The latter have many potential applications including spintronics and as networks for transmitting quantum information between processing nodes on a chip, for example. It is the latter application that is considered in this paper. Information in quantum spin networks is encoded in quantum states and its propagation is governed by the Schrodinger equation. Recent work has shown that this leads to new phenomena such as the emergence of anti-gravity centers for spin chains [1]

The dynamic behavior of even simple spin networks is complicated. For example, an excitation created at one end of a linear chain of spins, does not propagate in a classical fashion, hopping between neighboring spins from one end to the next. Rather the excitation creates a wavepacket, which is a superposition of eigenstates of the Hamiltonian, that evolves, dispersing and refocusing. Information transfer from one spin to another in a spin network is therefore not straightforward. Neglecting environmental decoherence, quantum transport is determined by the Hamiltonian of a system $H$ via the Schrodinger equation

$$
\imath \hbar \frac{\partial}{\partial t} \Psi(\mathbf{x}, t)=H \Psi(\mathbf{x}, t),
$$

where $\imath=\sqrt{-1}$ and $\hbar$ is the Planck constant divided by $2 \pi$. We shall choose units such that $\hbar=1$. Expressing $\Psi(\mathbf{x}, 0)$ as a linear combination of eigenfunctions $\phi_{n}(\mathbf{x})$ of the Hamiltonian,

$$
\Psi(\mathbf{x}, 0)=\sum_{n} c_{n} \phi_{n}(\mathbf{x})
$$

where $H \phi_{n}(\mathbf{x})=E_{n} \phi_{n}(\mathbf{x})$ and $E_{n}$ is a real number corresponding to the energy of $\phi_{n}(\mathbf{x})$, we see immediately that for a static Hamiltonian $H$

$$
\Psi(\mathbf{x}, t)=e^{-\imath H t} \Psi(\mathbf{x}, 0)=\sum_{n} c_{n} e^{-\imath E_{n} t} \phi_{n}(\mathbf{x}) .
$$

$\Psi(\mathrm{x}, t)$ governs the evolution of quantum states and the propagation of information encoded in it.

\section{QUANTUm SpIn Networks}

A quantum spin network for our purposes is simply a collection of $N$ spin- $\frac{1}{2}$ particles arranged in space with some of coupling between spins specified by an interaction Hamiltonian

$$
H=\sum_{m, n=1}^{N} J_{m n}\left(\sigma_{m}^{x} \sigma_{n}^{x}+\sigma_{m}^{y} \sigma_{n}^{y}+\epsilon \sigma_{m}^{z} \sigma_{n}^{z}\right) .
$$

$\epsilon$ is a constant that depends on the type of interaction with $\epsilon=0$ for XX coupling and $\epsilon=1$ for Heisenberg coupling, being common. $J_{m n}$ is the strength of the coupling between spin $m$ and spin $n$, usually proportional to the cubic power of the physical distance between the two spins. The factors $\sigma_{i}^{x, y, z}$ denote the single spin Pauli operators

$$
\sigma^{x}=\left(\begin{array}{ll}
0 & 1 \\
1 & 0
\end{array}\right), \quad \sigma^{y}=\left(\begin{array}{cc}
0 & -\imath \\
\imath & 0
\end{array}\right), \quad \sigma^{z}=\left(\begin{array}{cc}
1 & 0 \\
0 & -1
\end{array}\right) .
$$

$n$ indicates the position of the spin the operator is acting on

$$
\sigma_{n}^{x, y, z}=I_{2 \times 2} \otimes \ldots \otimes I_{2 \times 2} \otimes \sigma^{x, y, z} \otimes I_{2 \times 2} \otimes \ldots \otimes I_{2 \times 2},
$$

where the factor $\sigma^{x, y, z}$ occupies the $n$th position among the $N$ factors. The system Hilbert space on which $H$ acts is conveniently taken as $\mathcal{H}:=\mathbb{C}^{2^{N}}$.

We restrict our attention in this paper to the single excitation subspace, i.e., it is assumed that the total number of excitations in the network is one. The state space of the network is then spanned by the subset of $N$ single excitation quantum states $\{|n\rangle: n=1, \ldots, N\}$, where $|n\rangle=|\uparrow \uparrow \ldots \uparrow \downarrow \uparrow \ldots \uparrow \uparrow\rangle$ 
indicates that the excitation is localized at spin $n$. However, unlike in a classical network the system can be in any superposition of these basis states. The natural coupling among the spins allows an excitation at $n$ to drift towards an excitation at $m$, but the fidelity is limited by

$$
\begin{aligned}
p_{m n}(t) & =\left|\left\langle m\left|e^{-\imath H_{1} t}\right| n\right\rangle\right|^{2} \\
& =\left|\sum_{k=0}^{\tilde{N}}\left\langle m\left|\Pi_{k}\right| n\right\rangle e^{-\imath \lambda_{k} t}\right|^{2} \\
& \leq\left(\sum_{k=0}^{\tilde{N}}\left|\left\langle m\left|\Pi_{k}\right| n\right\rangle\right|\right)^{2}=: p_{m n}^{*},
\end{aligned}
$$

where $\Pi_{k}$ is the projector onto the $k$ th eigenspace of the Hamiltonian $H=\sum_{k} \lambda_{k} \Pi_{k}$ and the $\Pi_{k}, k=1, \ldots, N$, correspond to the single excitation subspace $\mathcal{H}_{1} \cdot p_{m n}^{*}$, also referred to as Information Transfer Capacity (ITC), is an upper bound on $p_{m n}(t)$, which is attainable if there exist a time $t \geq 0$ such that

$$
e^{-\imath \lambda_{k} t}=s_{k} e^{i \phi} \quad \forall k \text { s.t. } s_{k} \neq 0,
$$

where $s_{k}=\operatorname{Sgn}\left(\left\langle i\left|\Pi_{k}\right| j\right\rangle\right) \in\{0, \pm 1\}$ is a sign factor and $\phi$ an arbitrary global phase factor. Terms with $s_{k}=0$ correspond to eigenspaces that have no overlap with the initial or final state and can be ignored. Restricting ourselves to the set $K^{\prime}$ of indices for which $s_{k}= \pm 1$

$$
s_{k}=\exp \left[-\imath \pi\left(2 n_{k}+\frac{1}{2}\left(s_{k}-1\right)\right)\right] \quad \forall k \in K^{\prime}
$$

where $n_{k}$ is an integer. Inserting this into (6), taking the logarithm and dividing by $-\imath$

$$
\lambda_{k} t=2 \pi n_{k}+\frac{\pi}{2}\left(s_{k}-1\right)-\phi \quad \forall k \in K^{\prime} .
$$

To obtain meaningful constraints independent of the arbitrary phase $\phi$ we subtract the equations

$$
\left(\lambda_{k}-\lambda_{\ell}\right) t=2 \pi\left(n_{k}-n_{\ell}\right)+\frac{\pi}{2}\left(s_{k}-s_{\ell}\right) \quad \forall k, \ell \in K^{\prime} .
$$

These conditions are necessary and sufficient for attainability and physical, involving only differences of the eigenvalues, which are observable and independent of arbitrary phases. Broadly speaking the bounds are attainable in principle, i.e., we can get arbitrarily close to achieving the maximum transition probability simply by waiting the right amount of time $t$, if the transition frequencies $\omega_{1 k}$ are not rationally dependent.

In the context of spin chains an attainable upper bound of 1 for the transition probability $m \rightarrow n$ means that the network is capable for transferring an excitation from node $m$ to node $n$ with fidelity arbitrarily close to 1 . Previous work has shown that the maximum information transfer capacity between the end spins in a chain with uniform coupling between nearest neighbors is typically 1 regardless of the length of the chain. However, unlike in the classical case, the maximum transfer capacity from an end spin to a spin in the middle of the chain is far less than 1 . Thus, it is possible for an excitation to move from one end of the chain to the other without passing through the center.

\section{CONTROL OF INFORMATION TRANSFER}

The information transfer capacities of spin networks are interesting from a theoretical point of view in terms of understanding information flow in quantum networks and the restrictions resulting from quantum mechanical evolution. However, even if perfect state transfer between two nodes in a spin network is possible, in practice the time required to achieve a sufficiently high fidelity can be very long. For example, for a spin chain with only five nodes and uniform Heisenberg coupling, computation of the maximum information transfer capacity and attainability considerations show that state transfer from 1 to 5 can be achieved with arbitrarily high fidelity. However, when the transfer time is limited to say 1000 time steps in units of $J^{-1}$ then the attainable fidelity is only around $90 \%$. This is where control becomes relevant.

Previous work has shown that even bang-bang switching control of a local perturbation can significantly speed up information transfer between two ends of a chain with Heisenberg coupling [2]. The type of control employed in this work was very simple, requiring no more than bang-bang switching of a fixed local perturbation $H_{C}$ to the Hamiltonian so that the evolution of the system was governed by the original system Hamiltonian $H_{0}$ when the control was turned off, and by the perturbed Hamiltonian $H_{0}+H_{C}$ when the control was turned on. The control perturbations $H_{C}$ considered in [2] involved local perturbation of the coupling $J_{12}$ between the first pair of spins. As manipulating the coupling strength between spins can be difficult to achieve we can alternatively consider a perturbation that involves local detuning. Lie algebra considerations show that for a chain with Heisenberg coupling a simple detuning perturbation of the form $H_{C}=\sigma_{1}^{z}$ is sufficient for full controllability of the system on the single excitation subspace [3]. Optimizing the switching times as described in [2] shows that we can achieve high-fidelity state transfer. Fig. 1 shows an example of an optimal switching sequence and the corresponding transfer probability $p_{17}(t)$ as a function of time. We see that we can indeed achieve constructive interference or refocusing of the excitation at the target time at the desired end node. For the purposes of better illustration of the dynamical evolution we have chosen a short chain but the procedure is effective for chains with hundreds of nodes, although the resulting trajectories are extremely complex.

A perhaps even more interesting example for control is information transfer between nodes in a network beyond what is possible without control according to the maximum information transfer capacity. A simple example of this type is information transfer between nodes in a ring. For this arrangement of spins it can be shown that the transfer fidelity between nodes is limited and usually strictly less than 1 . In particular this is the case for information transfer from node 1 to 4 , for example, in a ring with seven nodes. In this case we can derive analytic expressions for the maximum information transfer capacity, which show that $p_{14}(t)$ is strictly less than 1 [4]. Unfortunately, unlike for chains, permutation symmetry of the nodes in a ring shows that applying a control of type $\sigma_{1}^{z}$ 

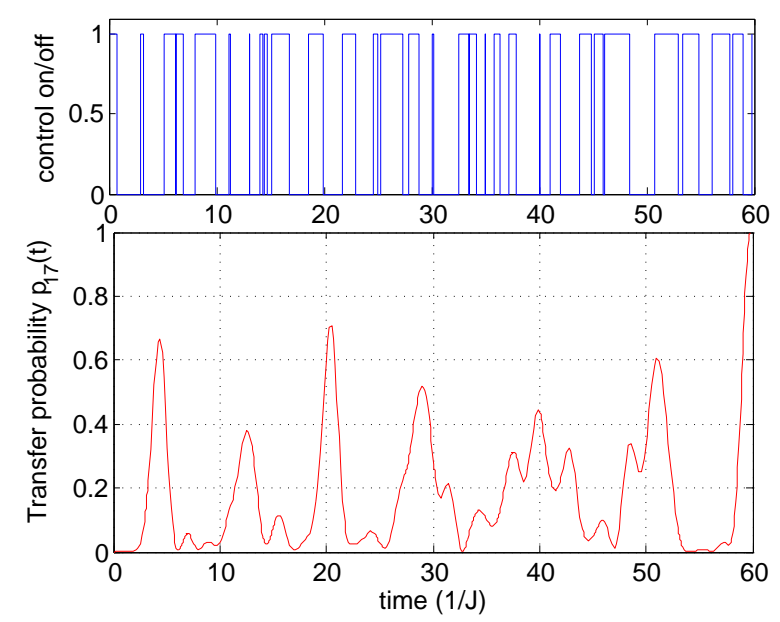

Fig. 1. Example of switching control and corresponding evolution of transfer probability $p_{17}(t)$ for Heisenberg spin chain of length 7 .

to the first spin is not sufficient for controllability. For example, the operation of swapping spins $k$ and $N+2-k$ commutes both with $H_{0}$ and $H_{C}$ and is therefore a symmetry. There are two further symmetries and the Lie algebra dimension is only 17, and numerical test of the optimization show that the attainable fidelity is limited, consistent with the dynamical constraints imposed by the symmetries and dark states [5].

An alternative to temporal on-off switching control is the application of spatially distributed static perturbations. In this case we use the spatial degrees of freedom to control the flow of information. We choose the control Hamiltonian to be $H=\operatorname{diag}\left(c_{1}, \ldots, c_{N}\right)$ and optimize the spatial biases $c_{n}$ to maximize the transfer fidelity from the initial node to the target node at a certain target time. We can choose a fixed target time $T$ and find $\mathbf{c}=\left(c_{n}\right)$ to maximize

$$
\left|\left\langle m\left|\exp \left[-\imath T\left(H_{0}+H_{C}(\mathbf{c})\right)\right]\right| n\right\rangle\right|^{2},
$$

or we can let $T$ vary within a certain range. An example of the resulting biases and evolution of the transfer probability for transfer from node 1 to node 4 and 5 , respectively, is shown in Fig. 2. The transfer fidelities are on the order of $1-10^{-4}$. The solutions are not unique. The top graph shows larger biases with less variation across nodes, which may be preferable from a practical point of view but has the disadvantage of resulting in faster oscillations. They are therefore less robust with regard to variations in the readout time. The bottom graph shows another solution with a large bias on one node, which suppresses rapid oscillations in the transfer probability $p_{15}(t)$ and suggests greater robustness with regard to readout mistiming.

\section{IDENTIFICATION OF SySTEM PARAMETERS}

The examples show that both types of control can be very effective with suitable optimization. However, the control design in both cases is model-based, and in practice the model parameters for spin networks will often at best be known
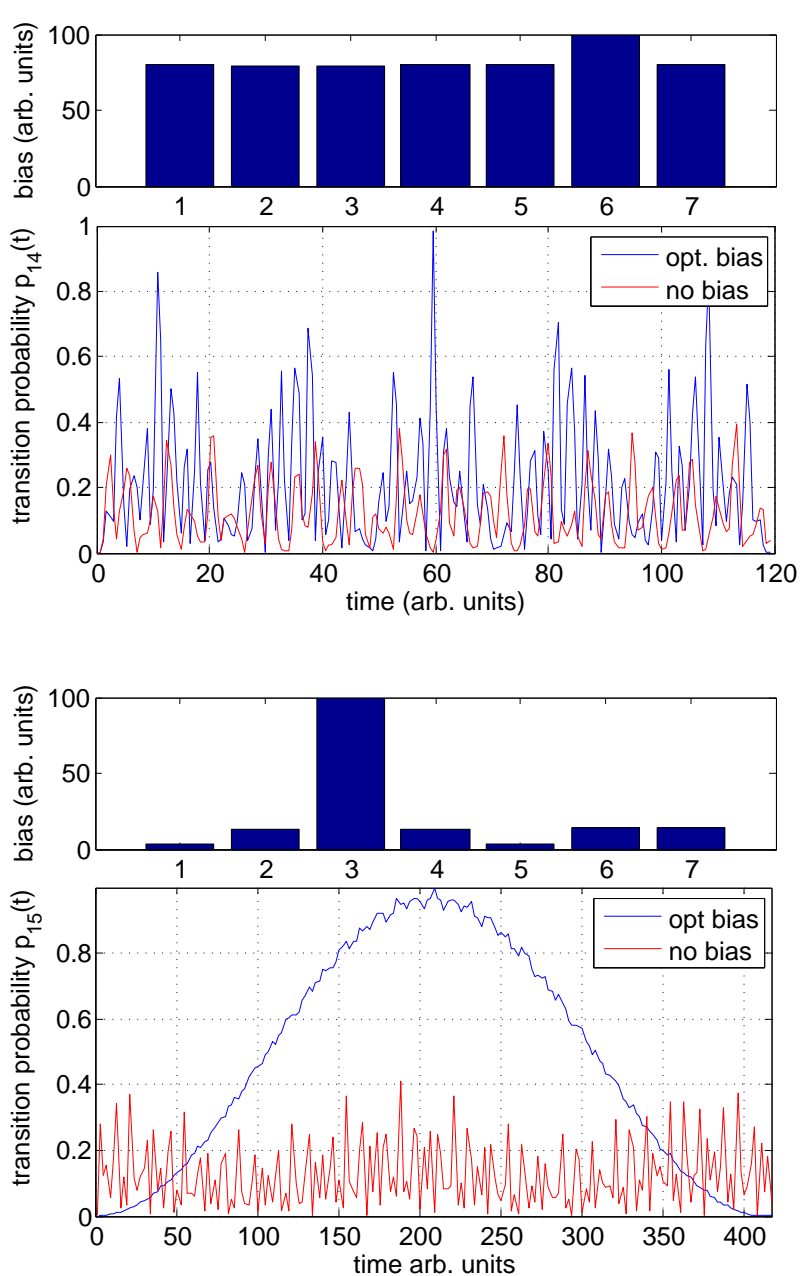

Fig. 2. Example of spatial bias control and corresponding evolution of transfer probability $p_{14}(t)$ (top) and $p_{15}(t)$ (bottom) for Heisenberg spin ring of length 7 . The red lines in the graph show the corresponding transfer probabilities without control.

approximately and may vary, in particular for engineered systems subject to fabrication tolerances. Previous work on control suggests reasonable robustness of optimal controls with regard to model uncertainties [2], but experimental system identification is expected to be crucial for the success of quantum control of engineered spin networks. In practice there are numerous uncertainties ranging from the network topology, to the precise number of spins in the network and the strength of the coupling between connected nodes.

System identification for quantum systems, including spin networks, has rapidly become a hot topic generating numerous papers [6] - [16]. However, these papers make numerous assumptions on the available resources, e.g., requiring prior knowledge of the precise topology, including the exact number of spins and high-precision quantum state tomography, resources and knowledge that may not be available in practice. In the following we consider a simple protocol to identify both the number of spins in the network and the coupling strength 
for rings with uniform coupling using a simple measurement protocol that requires only a small number of binary-outcome measurements on fixed spin in the network.

We assume uniform coupling strength and that we can initialize the system in state $|1\rangle$ and measure it in the same state $|1\rangle$ at time $t \in[0, T]$, resulting in a measurement outcome of 0 or 1 only. $T$ must be sufficiently large to capture the complete dynamics of this measurement trace. The Hamiltonian of the system is parametrized as an $N \times N$ matrix

$$
H_{1}(N, J)=\left(\begin{array}{ccccccccc}
0 & J & \ldots & 0 & 0 & 0 & \ldots & 0 & J \\
J & 0 & \ldots & 0 & 0 & 0 & \ldots & 0 & 0 \\
\vdots & \vdots & \ddots & \vdots & \vdots & \vdots & & \vdots & \vdots \\
0 & 0 & \ldots & 0 & J & 0 & \ldots & 0 & 0 \\
0 & 0 & \ldots & J & 0 & J & \ldots & 0 & 0 \\
0 & 0 & \ldots & 0 & J & 0 & \ldots & 0 & 0 \\
\vdots & \vdots & & \vdots & \vdots & \vdots & \ddots & \vdots & \vdots \\
0 & 0 & \ldots & 0 & 0 & 0 & \ldots & 0 & J \\
J & 0 & \ldots & 0 & 0 & 0 & \ldots & J & 0
\end{array}\right)
$$

The probability of measuring 1 at time $t$ is

$$
\theta(N, J, t)=\left|\left\langle 1\left|e^{-i H_{1}(N, J) t}\right| 1\right\rangle\right|^{2} .
$$

So given $M$ measurement results $E$ for times $t_{1}, \ldots, t_{m}$, each repeated $R_{m}$ times, the probability of $H_{1}(N, J)$ being the correct Hamiltonian is given by the binomial distribution

$$
\begin{gathered}
P\left(H_{1}(N, J) \mid E\right)= \\
\prod_{m}\left(\begin{array}{c}
A_{m} \\
R_{m}
\end{array}\right) \theta\left(N, J, t_{m}\right)^{A_{m}}\left(1-\theta\left(N, J, t_{m}\right)\left(t_{m}\right)\right)^{R_{m}-A_{m}}
\end{gathered}
$$

where $A_{m}$ is the number of 1 measurements at time $t_{m}$. For numerical reasons use instead the log likelihood

$$
L(N, J \mid E)=-\log P\left(H_{1}(N, J \mid E)\right) .
$$

To estimate the parameters $N$ and $J$, we iteratively take $M$ measurements at times $t_{m}$ sampled in the time interval $[0, T]$ using the Hammersley low-discrepancy sequence. We then sample $L$ over a given parameter domain $\left\{N_{\min }, N_{\min }+\right.$ $\left.1, \ldots, N_{\max }\right\} \times\left[J_{\min }, J_{\max }\right]$ with $K$ sample points per ringsize parameter $N$. Initially $K$ points $p_{k}$ are sampled uniformly for each size $N$ in the coupling strength parameter interval $\left[J_{\min }, J_{\max }\right] . L$ is evaluated at each sample point $p_{k}$. Then the $K$ samples are resampled according to the sampling density function

$$
D(j)=\frac{1}{2}\left(p_{k+1}-p_{k-1}\right) L(N, j \mid E) .
$$

where $k$ is chosen such that $p_{k}$ is closest to $j, p_{-1}=p_{0}=0$ and $p_{K+1}=p_{K}=T$ at the interval boundaries. Iteratively additional measurements are taken by continuing the Hammersley sequence on the measurement trace, updating the $L$ values at the sample points to update the log-likelihood for the
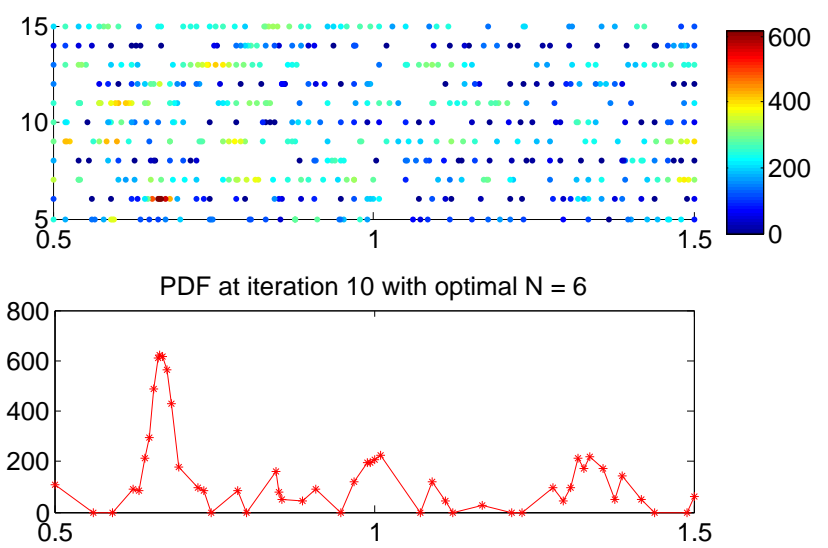

Fig. 3. Network identification example for a ring with 6 nodes and coupling strength 0.666 . Top: log-likelihood sample positions in the parameter domain $\{5,6, \ldots, 15\} \times[.5,1.5]$, after 10 iterations of taking 10 time samples, repeated 10 times, showing a clear peak close to the exact parameter values. Bottom: log-likelihood function over the coupling strength parameter domain $[.5,1.5]$ for $N=6$.

new measurements, and then resample the coupling strength intervals.

This is repeated until the sampled log-likelihood over the parameter domain has a clear peak according to the sample points taken. Then the $N$ value with the largest $L$ value for the sampled points is selected and a simple 1D maximization strategy, such as hill-climbing is used to find the coupling strength for which the log-likelihood is largest.

The samples per coupling strength interval $K$ can typically be small, say about 50 as the resampling process moves them towards the highest peaks in the interval and tracks these peaks. It is typically also sufficient to only take a few measurements $M$, say 10 per iteration, with a low number of repetitions $R$, say 10 . After a few iterations (about 10), the loglikelihood has a clear peak and ring size as well as coupling strength can then easily be estimated to high accuracy.

Fig. 3 shows an example for a ring with 6 spins and coupling strength 0.666. After 10 iterations, adding new sample times for the measurements, each measured 10 times, and then the final optimization for the coupling strength resulted in estimating the ring size clearly to be 6 with a coupling strength of 0.666083 , i.e. an error of $8.3 \times 10^{5}$.

\section{CONCLUSiON}

We have demonstrated a bang-bang and a bias control scheme for optimizing the information transfer between nodes in simple quantum spin networks, either ensuring maximal transfer in limited time or enabling maximal transfer against the natural dynamics of the network. We have further presented a scheme to simultaneously estimate the network size and coupling strength for simple networks with uniform coupling using an efficient sampling strategy to take measurements as well as sampling the parameter domain. Future work 
will extend the control and characterization schemes to more general and realistic quantum networks.

\section{REFERENCES}

[1] E. Jonckheere, S. G. Schirmer, F. C. Langbein, Geometry and curvature of spin networks, Control Applications (CCA), 2011 IEEE International Conference on, 786-791 (2011).

[2] S. G. Schirmer and P. J. Pemberton-Ross, Fast high-fidelity information transmission through spin-chain quantum wires, Phys. Rev. A 80(3), 030301 (2009).

[3] X. Wang, P. J. Pemberton-Ross and S. G. Schirmer, Symmetry and subspace controllability for spin networks with a single-node control, IEEE Trans. Autom. Control 57(8), 1945-1956 (2012).

[4] E. Jonckheere, F. C. Langbein, S. G. Schirmer, "Curvature of quantum rings," Communications Control and Signal Processing (ISCCSP), 2012 5th International Symposium on, 1-6 (2012).

[5] P. J. Pemberton-Ross, A. Kay and S. G. Schirmer, "Quantum control theory for state transformations: Dark states and their enlightenment," Phys. Rev. A 82(4), 042322 (2010).

[6] S. G. Schirmer, A. Kolli, D. K.L. Oi, Experimental Hamiltonian identification for controlled two-level systems, Phys. Rev. A 69, 050306(R) (2004).

[7] S. G. Schirmer, A. Kolli, D. K. L. Oi, J. H. Cole, Experimental Hamiltonian identification for Qubits subject to multiple independent control mechanisms, In: Proc. 7th Int. Conf. QCMC, Glasgow 25-29 July 2004 (AIP 2004)

[8] J. H. Cole et al. Identifying an experimental two-state Hamiltonian to arbitrary accuracy, Phys. Rev. A 71, 062312 (2005).

[9] S. G. Schirmer and D. K. L. Oi, Two-Qubit Hamiltonian Tomography by Bayesian Analysis of Noisy Data Phys. Rev. A 80, 022333 (2009)

[10] J. H. Cole et al. Identifying a Two-State Hamiltonian in the Presence of Decoherence, Phys. Rev. A 73, 062333 (2006).

[11] S. J. Devitt et al. Subspace Confinement: How good is your qubit?, New J. Phys. 9, 384 (2007)

[12] S. G. Schirmer, D. K. L. Oi and S. J. Devitt, Physics-based mathematica models for quantum devices via experimental system identification, J. Phys.: Conf. Series 107012011 (2008)

[13] S. G. Schirmer and D. K. L. Oi, Quantum System Identification by Bayesian Analysis of Noisy Data: Beyond Hamiltonian Tomography Laser physics 20 (5), 1203-1209 (2010)

[14] D. Burgarth, K. Maruyama, F. Nori, Coupling strength estimation for spin chains despite restricted access, Phys. Rev. A 79, 020305(R) (2009)

[15] D. K. L. Oi and S. G. Schirmer, Quantum system characterization with limited resources, Phil. Trans. Royal. Soc. A 370, 5386-5395 (2012)

[16] Daniel Burgarth, Koji Maruyama, Indirect Hamiltonian Identification through a small gateway, New J. Phys. 11, 103019 (2009)

[17] H. Niederreiter, Random Number Generation and Quasi-Monte Carlo Methods (SIAM Review, 1992)

[18] J. A. Quinn, F. C. Langbein, R. R. Martin, G. Elber, Density-Controlled Sampling of Parametric Surfaces Using Adaptive Space-Filling Curves. Springer LNCS 4077, 465-484 (2006). 